\title{
The semistar operations on certain Prüfer domain, II
}

\author{
Ryûki MATSUDA*
}

\begin{abstract}
Let $D$ be a 1-dimensional Prüfer domain with exactly two maximal ideals. We completely determine the star operations and the semistar operations on $D$.
\end{abstract}

Let $G$ be a torsion-free abelian additive group. If $G$ is not discrete, $G$ is called indiscrete. If every non-empty subset $S$ of $G$ which is bounded below has its infimum $\inf (S)$ in $G$, then $G$ is called complete. If $G$ is not complete, $G$ is called incomplete. Let $D$ be a 1-dimensional Prüfer domain with exactly two maximal ideals $M$ and $N$, let $V=D_{M}$ (resp., $W=D_{N}$ ), let $v$ (resp., $w$ ) be a valuation belonging to $V$ (resp., $W$ ), let $\Gamma$ (resp., $\Gamma^{\prime}$ ) be the value group of $v$ (resp., $w$ ), and let $K$ be the quotient field of $D$. Let $\Sigma(D)$ (resp., $\Sigma^{\prime}(D)$ ) be the set of star operations (resp., semistar operations) on $D$. In [1, Proposition $6(2)]$, we proved that, if both $\Gamma$ and $\Gamma^{\prime}$ are discrete, then $|\Sigma(D)|=1$ and $\left|\Sigma^{\prime}(D)\right|=7$. In this paper, we prove the following,

Theorem Let $D$ be a 1-dimensional Prüfer domain with exactly two maximal ideals $M$ and $N$, let $\Gamma$ (resp., $\Gamma^{\prime}$ ) be the value group belonging to the valuation ring $D_{M}$ (resp., $\left.D_{N}\right)$.

(1) If both $\Gamma$ and $\Gamma^{\prime}$ are discrete, then $|\Sigma(D)|=1$ and $\left|\Sigma^{\prime}(D)\right|=7$.

(2) If $\Gamma$ is discrete, and if $\Gamma^{\prime}$ is indiscrete, then $|\Sigma(D)|=2$ and $\left|\Sigma^{\prime}(D)\right|=14$.

(3) If both $\Gamma$ and $\Gamma^{\prime}$ are indiscrete, then $|\Sigma(D)|=4$ and $\left|\Sigma^{\prime}(D)\right|=30$.

In the above Theorem, (1) was proved in [1, Proposition 6 (2)] as was mentioned. (2) was proved in [2] in the case where $\Gamma^{\prime}$ is incomplete. (3) was studied in [2] in the case where both $\Gamma$ and $\Gamma^{\prime}$ are incomplete. Unfortunately, [2, Theorem (4)] and its proof were incomplete. Section 1 of this paper contains preliminary results, and in Section 2, we prove Theorem (3). The proof of Theorem (2) is similar to that of $[2$, Theorem (2)].

Throughout the paper, we confer [2], $p$ denotes an element of $M \backslash N, q$ denotes an element of $N \backslash M$, and $x$ denotes an element of $K \backslash\{0\}$.

Received 8 August 2013; revised 24 October 2013

2000 Mathematics Subject Classification. Primary 13A15

Key Words and Phrases. star operation, semistar operation.

*Professor Emeritus, Ibaraki University, Mito, Ibaraki 310-8512, Japan (rmazda@adagio.ocn.ne.jp) 


\section{$\S 1 \quad$ Preliminary results}

The set of non-zero fractional ideals of $D$ is denoted by $\mathrm{F}(D)$, and the set of non-zero $D$-submodules of $K$ is denoted by $\overline{\mathrm{F}}(D)$.

(1.1) Let $x \in K \backslash\{0\}$ and let $I \in \overline{\mathrm{F}}(D)$. Then $x \in I$ if and only if $v(x) \in v(I)$ and $w(x) \in w(I)$.

(1.2) $([2,(1.1)]) \quad$ (1) Each element $x \in D \backslash\{0\}$ can be expressed as 1 or $p$ or $q$ or $p q$ uniquely up to associates, where $p \in M \backslash N$ and $q \in N \backslash M$.

(2) Each element $x \in K \backslash\{0\}$ can be expressed as 1 or $p$ or $q$ or $p q$ or $\frac{1}{p}$ or $\frac{1}{q}$ or $\frac{q}{p}$ or $\frac{p}{q}$ or $\frac{1}{p q}$ uniquely up to associates.

An ideal $A_{0}$ of $D$ is called with type $\alpha$, if there exists a subset $\left\{p_{\lambda} \mid \lambda \in \Lambda\right\}$ of $M \backslash N$ so that $A_{0}$ is generated by the set $\left\{p_{\lambda} \mid \lambda \in \Lambda\right\}$, and there does not exist inf $v\left(A_{0}\right)$. An ideal $B_{0}$ is called with type $\beta$, if there exists a subset $\left\{q_{\sigma} \mid \sigma \in \Sigma\right\}$ of $N \backslash M$ so that $B_{0}$ is generated by the set $\left\{q_{\sigma} \mid \sigma \in \Sigma\right\}$, and there does not exist inf $w\left(B_{0}\right)$. If $\Gamma$ (resp., $\Gamma^{\prime}$ ) is discrete, then $\Gamma$ (resp., $\Gamma^{\prime}$ ) is complete. If $\Gamma$ (resp., $\Gamma^{\prime}$ ) is complete, then there does not exist an ideal of $D$ with type $\alpha$ (resp., $\beta$ ). Throughout the paper, $A_{0}, A_{1}, \cdots$ (resp., $\left.B_{0}, B_{1}, \cdots\right)$ denote ideals of $D$ with type $\alpha$ (resp., $\beta$ ). The definition of type $\alpha$ (resp., $\beta$ ) is simpler than that in [2], and both are essentially the same.

Let $I, J \in \overline{\mathrm{F}}(D)$. If $I=x J$ for some $x \in K \backslash\{0\}$, we say that $I$ and $J$ are similar.

(1.3) (1) $\mathrm{F}(D)=\left\{(x), x M, x N, x A_{0}, x B_{0}, x M N, x A_{0} N, x B_{0} M, x A_{0} B_{0}, K \mid x \in\right.$ $K \backslash\{0\}, A_{0}$ (resp., $B_{0}$ ) is an ideal with type $\alpha$ (resp., $\beta$ ) $\}$.

(2) $D, M, N, A_{0}, B_{0}, M N, A_{1} N, B_{1} M, A_{2} B_{2}$ are not similar with each other.

(3) We have $A_{0}=A_{0}^{\mathrm{v}}, B_{0}=B_{0}^{\mathrm{v}}$ and $A_{0} B_{0}=\left(A_{0} B_{0}\right)^{\mathrm{v}}$.

(1.4) Example. Let $G_{1}$ (resp., $G_{2}$ ) be non-zero torsion-free abelian additive groups. Let $G_{1} \oplus G_{2}$ be their direct sum with the lexicographic order: Let $x:=$ $\left(a_{1}, a_{2}\right), y:=\left(b_{1}, b_{2}\right)$ be elements of $G_{1} \oplus G_{2}$ with $a_{2}<b_{2}$, then let $x<y$. Let $v_{0}$ (resp., $w_{0}$ ) be the projection mapping of $G_{1} \oplus G_{2}$ to $G_{1}$ (resp., $G_{2}$ ). Let $k$ be a field, and let $K$ be the quotient field of the semigroup ring $k\left[X ; G_{1} \oplus G_{2}\right]$. Let $v$ (resp., $w$ ) be the canonical extension of $v_{0}$ (resp., $w_{0}$ ) to a valuation on $K$, and let $V$ (resp., $W$ ) be the valuation ring on $K$ belonging to $v$ (resp., $w$ ), and let $M^{\prime}$ (resp., $N^{\prime}$ ) be the maximal ideal of $V$ (resp., $W$ ). Let $D:=V \cap W$, and let $M:=M^{\prime} \cap D$ and $N:=N^{\prime} \cap D$. Then we have $V=D_{M}, M^{\prime}=M V, W=D_{N}$ and $N^{\prime}=N W$. We have $\Gamma=G_{1}$, and $\Gamma^{\prime}=G_{2}$.

(1.5) Let $\star$ be a star operation.

If $N^{\star}=D$, then $\left(A_{0} N\right)^{\star}=A_{0}$.

If $N^{\star}=N$, then $\left(A_{0} N\right)^{\star}=A_{0} N$.

If $M^{\star}=D$, then $\left(B_{0} M\right)^{\star}=B_{0}$.

If $M^{\star}=M$, then $\left(B_{0} M\right)^{\star}=B_{0} M$. 
(1.6) (1) $x M V \subseteq M$ is impossible.

$x M V \subseteq N$ is impossible.

$x N W \subseteq M$ is impossible.

$x N W \subseteq N$ is impossible.

(2) $x M V \subseteq W$ is impossible.

$x N W \subseteq V$ is impossible.

Let $I^{\mathrm{d}^{\prime}}=I$ (resp., $I^{\mathrm{v}^{\prime}}=\left(I^{-1}\right)^{-1}, I^{\mathrm{e}}=K$ ) for each $I \in \overline{\mathrm{F}}(D)$. Then $\mathrm{d}^{\prime}$ (resp., $\mathrm{v}^{\prime}, \mathrm{e}$ ) is a semistar operation on $D$, and is called the $\mathrm{d}^{\prime}$-operation (resp., $\mathrm{v}^{\prime}$-operation, e-operation) on $D$.

(1.7) $V^{\mathrm{v}^{\prime}}=K, W^{\mathrm{v}^{\prime}}=K$.

Let $I \subseteq J$ be an inclusion relation of elements of $\overline{\mathrm{F}}(D)$. A mapping $\star$ from $\overline{\mathrm{F}}(D)$ to $\overline{\mathrm{F}}(D)$ is called monotone for $I \subseteq J$, if $I^{\star} \subseteq J^{\star}$.

(1.8) (cf., $[2,(1.8)]) \quad$ Let $\star$ be a semistar operation on $D$.

(1) $V^{\star}$ is either $V$ or $K . W^{\star}$ is either $W$ or $K$.

(2) $(M V)^{\star}$ is either $M V$ or $V$ or $K .(N W)^{\star}$ is either $N W$ or $W$ or $K$.

(3) If $V^{\star}=V$, then $\left(A_{0} V\right)^{\star}=A_{0} V$ for every ideal $A_{0}$ of $D$ with type $\alpha$. If $W^{\star}=W$, then $\left(B_{0} W\right)^{\star}=B_{0} W$ for every ideal $B_{0}$ of $D$ with type $\beta$.

\section{$\S 2 \quad \Gamma$ and $\Gamma^{\prime}$ are indiscrete}

Throughut the section, we assume that $\Gamma$ and $\Gamma^{\prime}$ are indiscrete.

(2.1) (1) $M^{\mathrm{v}}=D, N^{\mathrm{v}}=D,(M N)^{\mathrm{v}}=D$.

(2) $\left(A_{0} N\right)^{\mathrm{v}}=A_{0},\left(B_{0} M\right)^{\mathrm{v}}=B_{0}$.

(3) $\left\{I \in \mathrm{F}(D) \mid I \varsubsetneqq I^{\mathrm{v}}\right\}=\left\{x M, x N, x M N, x A_{0} N, x B_{0} M \mid x \in K \backslash\{0\}, A_{0}\right.$ (resp., $\left.B_{0}\right)$ is an ideal with type $\alpha$ (resp., $\left.\left.\beta\right)\right\}$.

(2.2) Let $x \in K \backslash\{0\}$ and let $A_{0}, A_{1}$ (resp., $B_{0}, B_{1}$ ) be an ideal of $D$ with type $\alpha$ (resp., $\beta$ ).

(1) If $x M \subseteq D$, then $(x) \subseteq D$.

If $x M \subseteq M$, then $(x) \subseteq D$.

If $x M \subseteq N$, then $(x) \subseteq N$.

If $x M \subseteq A_{0}$, then $(x) \subseteq A_{0}$.

If $x M \subseteq B_{0}$, then $(x) \subseteq B_{0}$.

If $x M \subseteq M N$, then $(x) \subseteq N$.

If $x M \subseteq A_{0} N$, then $(x) \subseteq A_{0} N$.

If $x M \subseteq B_{0} M$, then $(x) \subseteq B_{0}$.

If $x M \subseteq A_{0} B_{0}$, then $(x) \subseteq A_{0} B_{0}$.

(2) If $x N \subseteq D$, then $(x) \subseteq D$.

If $x N \subseteq M$, then $(x) \subseteq M$.

If $x N \subseteq N$, then $(x) \subseteq D$. 


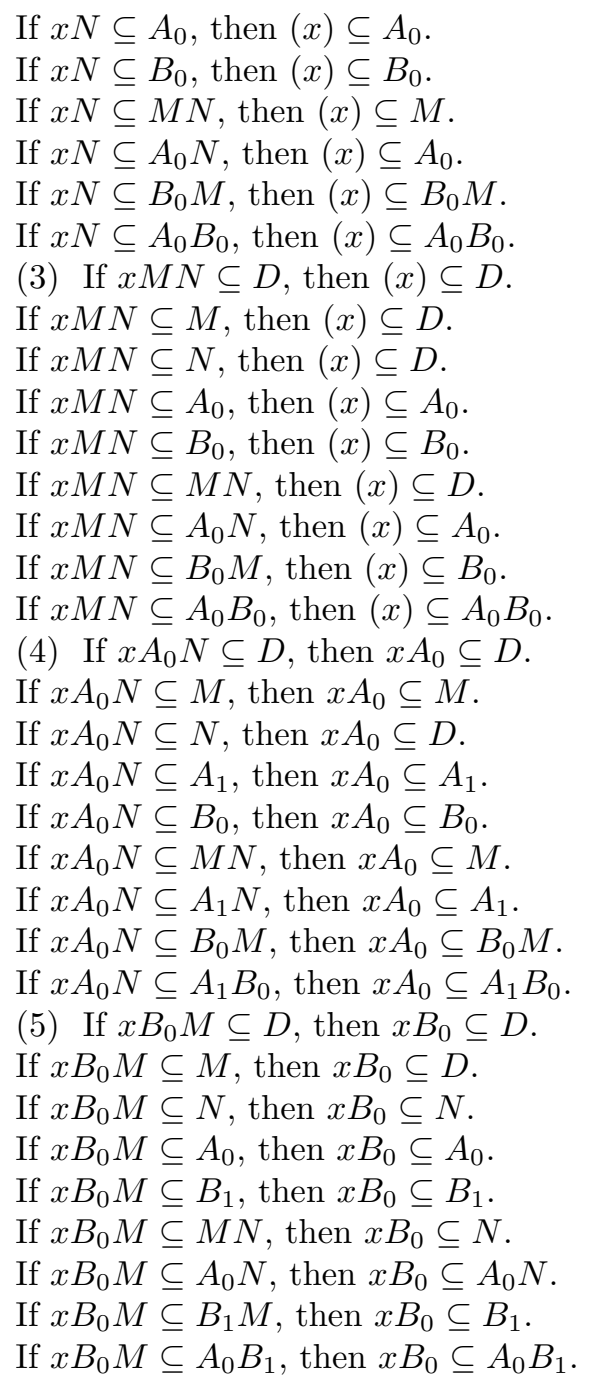

(2.3) (1) Set $M^{\star_{1}}:=M, N^{\star_{1}}:=N$. Then there is a unique star operation $\star_{1}$, and $\star_{1}=\mathrm{d}$.

(2) Set $M^{\star_{2}}:=M, N^{\star_{2}}:=D$. Then there is a unique star operation $\star_{2}$.

(3) Set $M^{\star_{3}}:=D, N^{\star_{3}}:=N$. Then there is a unique star operation $\star_{3}$.

(4) Set $M^{\star_{4}}:=D, N^{\star_{4}}:=D$. Then there is a unique star operation $\star_{4}$, and $\star_{4}=\mathrm{v}$.

(2.4) We have $|\Sigma(D)|=4$ and $\Sigma(D)=\left\{\star_{1}, \star_{2}, \star_{3}, \star_{4}\right\}$.

(2.5) (1) $\overline{\mathrm{F}}(D) \backslash \mathrm{F}(D)=\left\{x V, x W, x M V, x N W, x A_{0} V, x B_{0} W, K \mid x \in K \backslash\{0\}, A_{0}\right.$ (resp., $B_{0}$ ) is an ideal with type $\alpha$ (resp., $\beta$ ) $\}$.

(2) $V, W, M V, N W, A_{0} V, B_{0} W, K$ are not similar with each other. 
(2.6) Let $\star^{1}, \star^{2}$ be semistar operations on $D$ satisfying the followings:

(1) The restriction $\star_{1}^{\prime}:=\left.\star^{1}\right|_{F(D)}$ (resp., $\left.\star_{2}^{\prime}:=\left.\star^{2}\right|_{F(D)}\right)$ of $\star^{1}$ (resp., $\left.\star^{2}\right)$ to $\mathrm{F}(D)$ is a star operation on $D$, and $\star_{1}^{\prime}=\star_{2}^{\prime}$.

(2) We have that $V^{\star^{1}}=V^{\star^{2}}, W^{\star^{1}}=W^{\star^{2}},(M V)^{\star^{1}}=(M V)^{\star^{2}}$ and $(N W)^{\star^{1}}=$ $(N W)^{\star^{2}}$

Then we have $\star^{1}=\star^{2}$.

In the following (2.7), let $\star:=\star_{i}^{j}$. Since $\star_{i}$ is a star operation on $D$, we have $(x I)^{\star}=x I^{\star}$ for every $I \in \mathrm{F}(D)$. We define $(x V)^{\star}:=x V^{\star},(x W)^{\star}:=x W^{\star},(x M V)^{\star}:=$ $x(M V)^{\star},(x N W)^{\star}:=x(N W)^{\star}$ and $K^{\star}:=K$. We dfine $\left(A_{0} V\right)^{\star}:=A_{0} V$ (resp., $\left.\left(A_{0} V\right)^{\star}:=K\right)$ if $V^{\star}=V\left(\right.$ resp., $\left.V^{\star}=K\right),\left(B_{0} W\right)^{\star}:=B_{0} W$ (resp., $\left.\left(B_{0} W\right)^{\star}:=K\right)$ if $W^{\star}=W\left(\right.$ resp., $\left.W^{\star}=K\right),\left(x A_{0} V\right)^{\star}:=x\left(A_{0} V\right)^{\star}$, and $\left(x B_{0} W\right)^{\star}:=x\left(B_{0} W\right)^{\star}$.

(2.7) $\quad$ Let $1 \leq i \leq 4$.

(1) Set $I_{0}^{\star_{i}^{1}}:=I_{0}^{\star_{i}}$ for each $I_{0} \in \mathrm{F}(D)$, and set $V^{\star_{i}^{1}}:=V, W^{\star_{i}^{1}}:=W,(M V)^{\star_{i}^{1}}:=$ $M V$ and $(N W)^{\star_{i}^{1}}:=N W$. Then there is determined a unique mapping $\star_{i}^{1}$ from $\overline{\mathrm{F}}(D)$ to $\overline{\mathrm{F}}(D)$.

(2) Set $I_{0}^{\star_{i}^{2}}:=I_{0}^{\star_{i}}$ for each $I_{0} \in \mathrm{F}(D)$, and set $V^{\star_{i}^{2}}:=V, W^{\star_{i}^{2}}:=W,(M V)^{\star_{i}^{2}}:=$ $M V$ and $(N W)^{\star_{i}^{2}}:=W$. Then there is determined a unique mapping $\star_{i}^{2}$ from $\overline{\mathrm{F}}(D)$ to $\overline{\mathrm{F}}(D)$.

(3) Set $I_{0}^{\star_{i}^{3}}:=I_{0}^{\star_{i}}$ for each $I_{0} \in \mathrm{F}(D)$, and set $V^{\star_{i}^{3}}:=V, W^{\star_{i}^{3}}:=W,(M V)^{\star_{i}^{3}}:=V$ and $(N W)^{\star_{i}^{3}}:=N W$. Then there is determined a unique mapping $\star_{i}^{3}$ from $\overline{\mathrm{F}}(D)$ to $\overline{\mathrm{F}}(D)$.

(4) Set $I_{0}^{\star_{i}^{4}}:=I_{0}^{\star_{i}}$ for each $I_{0} \in \mathrm{F}(D)$, and set $V^{\star_{i}^{4}}:=V, W^{\star_{i}^{4}}:=W,(M V)^{\star_{i}^{4}}:=V$ and $(N W)^{\star_{i}^{4}}:=W$. Then there is determined a unique mappping $\star_{i}^{4}$ from $\overline{\mathrm{F}}(D)$ to $\overline{\mathrm{F}}(D)$.

(5) Set $I_{0}^{\star_{i}^{5}}:=I_{0}^{\star_{i}}$ for each $I_{0} \in \mathrm{F}(D)$, and set $V^{\star_{i}^{5}}:=V, W^{\star_{i}^{5}}:=K$ and $(M V)^{\star_{i}^{5}}:=$ $M V$. Then there is determined a unique mapping $\star_{i}^{5}$ from $\overline{\mathrm{F}}(D)$ to $\overline{\mathrm{F}}(D)$.

(6) Set $I_{0}^{\star_{i}^{6}}:=I_{0}^{\star_{i}}$ for each $I_{0} \in \mathrm{F}(D)$, and set $V^{\star_{i}^{6}}:=V, W^{\star_{i}^{6}}:=K$ and $(M V)^{\star_{i}^{6}}:=$ $V$. Then there is determined a unique mapping $\star_{i}^{6}$ from $\overline{\mathrm{F}}(D)$ to $\overline{\mathrm{F}}(D)$.

(7) Set $I_{0}^{\star_{i}^{7}}:=I_{0}^{\star_{i}}$ for each $I_{0} \in \mathrm{F}(D)$, and set $V^{\star_{i}^{7}}:=K, W^{\star_{i}^{7}}:=W$ and $(N W)^{\star_{i}^{7}}:=N W$. Then there is determined a unique mapping $\star_{i}^{7}$ from $\overline{\mathrm{F}}(D)$ to $\overline{\mathrm{F}}(D)$.

(8) Set $I_{0}^{\star_{i}^{8}}:=I_{0}^{\star_{i}}$ for each $I_{0} \in \mathrm{F}(D)$, and set $V^{\star_{i}^{8}}:=K, W^{\star_{i}^{8}}:=W$ and $(N W)^{\star_{i}^{8}}:=W$. Then there is determined a unique mapping $\star_{i}^{8}$ from $\overline{\mathrm{F}}(D)$ to $\overline{\mathrm{F}}(D)$.

(9) Set $I_{0}^{\star_{i}^{9}}:=I_{0}^{\star_{i}}$ for each $I_{0} \in \mathrm{F}(D)$, and set $V^{\star_{i}^{9}}:=K$ and $W^{\star_{i}^{9}}:=K$. Then there is determined a unique mapping $\star_{i}^{9}$ from $\overline{\mathrm{F}}(D)$ to $\overline{\mathrm{F}}(D)$.

(2.8) Each $\star_{i}^{j}$ satisfies the following conditions:

(1) $(x I)^{\star}=x I^{\star}$ for every $x \in K \backslash\{0\}$ and $I \in \overline{\mathrm{F}}(D)$;

(2) $I \subseteq I^{\star}$ for every $I \in \overline{\mathrm{F}}(D)$;

(3) $\left(I^{\star}\right)^{\star}=I^{\star}$ for every $I \in \overline{\mathrm{F}}(D)$. 
(2.9) Let $x \in K \backslash\{0\}, A_{0}, A_{1}$ be ideals of $D$ with type $\alpha$, and $B_{0}, B_{1}$ be ideals with type $\beta$.

(1) If $x M \subseteq V$, then $(x) \subseteq V$.

If $x M \subseteq W$, then $(x) \subseteq W$.

If $x M \subseteq M V$, then $(x) \subseteq V$.

If $x M \subseteq N W$, then $(x) \subseteq N W$.

If $x M \subseteq A_{0} V$, then $(x) \subseteq A_{0} V$.

If $x M \subseteq B_{0} W$, then $(x) \subseteq B_{0} W$.

(2) If $x N \subseteq V$, then $(x) \subseteq V$.

If $x N \subseteq W$, then $(x) \subseteq W$.

If $x N \subseteq M V$, then $(x) \subseteq M V$.

If $x N \subseteq N W$, then $(x) \subseteq W$.

If $x N \subseteq A_{0} V$, then $(x) \subseteq A_{0} V$.

If $x N \subseteq B_{0} W$, then $(x) \subseteq B_{0} W$.

(3) If $x M N \subseteq V$, then $(x) \subseteq V$.

If $x M N \subseteq W$, then $(x) \subseteq W$.

If $x M N \subseteq M V$, then $(x) \subseteq V$.

If $x M N \subseteq N W$, then $(x) \subseteq W$.

If $x M N \subseteq A_{0} V$, then $(x) \subseteq A_{0} V$.

If $x M N \subseteq B_{0} W$, then $(x) \subseteq B_{0} W$.

(4) If $x A_{0} N \subseteq V$, then $x A_{0} \subseteq V$.

If $x A_{0} N \subseteq W$, then $(x) \subseteq W$.

If $x A_{0} N \subseteq M V$, then $x A_{0} \subseteq M V$.

If $x A_{0} N \subseteq N W$, then $(x) \subseteq W$.

If $x A_{0} N \subseteq A_{1} V$, then $x A_{0} \subseteq A_{1} V$.

If $x A_{0} N \subseteq B_{0} W$, then $x A_{0} \subseteq B_{0} W$.

(5) If $x B_{0} M \subseteq V$, then $(x) \subseteq V$.

If $x B_{0} M \subseteq W$, then $x B_{0} \subseteq W$.

If $x B_{0} M \subseteq M V$, then $(x) \subseteq V$.

If $x B_{0} M \subseteq N W$, then $x B_{0} \subseteq N W$.

If $x B_{0} M \subseteq A_{0} V$, then $(x) \subseteq A_{0} V$.

If $x B_{0} M \subseteq B_{1} W$, then $x B_{0} \subseteq B_{1} W$.

(6) If $x V \subseteq V$, then $(x) \subseteq V$.

If $x V \subseteq M V$, then $(x) \subseteq M V$.

If $x V \subseteq A_{0} V$, then $(x) \subseteq A_{0} V$.

(7) If $x W \subseteq W$, then $(x) \subseteq W$.

If $x W \subseteq N W$, then $(x) \subseteq N W$.

If $x W \subseteq B_{0} W$, then $(x) \subseteq B_{0} W$.

(8) If $x M V \subseteq V$, then $(x) \subseteq V$.

If $x M V \subseteq M V$, then $(x) \subseteq V$.

If $x M V \subseteq A_{0} V$, then $(x) \subseteq A_{0} V$.

(9) If $x N W \subseteq W$, then $(x) \subseteq W$.

If $x N W \subseteq N W$, then $(x) \subseteq W$.

If $x N W \subseteq B_{0} W$, then $(x) \subseteq B_{0} W$. 
(2.10) (1) $\star_{3}^{1}, \star_{4}^{1}, \star_{3}^{2}, \star_{4}^{2}, \star_{3}^{5}, \star_{4}^{5}$ is not monotone for $M \subseteq M V$.

(2) $\star_{2}^{1}, \star_{4}^{1}, \star_{2}^{3}, \star_{4}^{3}, \star_{2}^{7}, \star_{4}^{7}$ is not monotone for $N \subseteq N W$.

(2.11) Set $\mathcal{I}:=\left\{M, N, M N, A_{0} N, B_{0} M, V, W, M V, N W \mid A_{0}\right.$ (resp., $B_{0}$ ) is an ideal with type $\alpha$ (resp., $\beta$ ) $\}$ and $\mathcal{J}:=\left\{V, W, M V, N W, A_{1} V, B_{1} W \mid A_{1}\right.$ (resp., $B_{1}$ ) is an ideal with type $\alpha$ (resp., $\beta$ ) $\}$. The followings are equivalent.

(1) $\star_{i}^{j}$ is a semistar operation.

(2) $\star_{i}^{j}$ is monotone for every $x I \subseteq J$, where $I \in \mathcal{I}$ and $J \in \mathcal{J}$.

Proof. Assume (2). We confer (1.6) and (1.8). Let $\star:=\star_{i}^{j}$, and let $x B_{0} W \subseteq J$ for some $J \in \overline{\mathrm{F}}(D)$.

The case $W^{\star}=W$. Then $\left(B_{0} W\right)^{\star}=B_{0} W$. Hence $\star$ is monotone for $x B_{0} W \subseteq J$.

The case $W^{\star}=K$. We may assume $J \in\left\{W, N W, B_{1} W, K\right\}$, where $B_{1}$ is with type $\beta$. Hence $J^{\star}=K$. Hence $\star$ is monotone for $x B_{0} W \subseteq J$.

(2.12)

$\star_{1}^{1}$ is a semistar operation on $D$, and $\star_{1}^{1}=\mathrm{d}^{\prime}$.

$\star_{1}^{2}$ is a semistar operation on $D$.

$\star_{2}^{2}$ is a semistar operation on $D$.

$\star_{1}^{3}$ is a semistar operation on $D$.

$\star_{3}^{3}$ is a semistar operation on $D$.

$\star_{1}^{4}$ is a semistar operation on $D$.

$\star_{2}^{4}$ is a semistar operation on $D$.

$\star_{3}^{4}$ is a semistar operation on $D$.

$\star_{4}^{4}$ is a semistar operation on $D$.

$\star_{1}^{5}$ is a semistar operation on $D$.

$\star_{2}^{5}$ is a semistar operation on $D$.

$\star_{1}^{6}$ is a semistar operation on $D$.

$\star_{2}^{6}$ is a semistar operation on $D$.

$\star_{3}^{6}$ is a semistar operation on $D$.

$\star_{4}^{6}$ is a semistar operation on $D$.

$\star_{1}^{7}$ is a semistar operation on $D$.

$\star_{3}^{7}$ is a semistar operation on $D$.

$\star_{1}^{8}$ is a semistar operation on $D$.

$\star_{2}^{8}$ is a semistar operation on $D$.

$\star_{3}^{8}$ is a semistar operation on $D$.

$\star_{4}^{8}$ is a semistar operation on $D$.

$\star_{1}^{9}$ is a semistar operation on $D$.

$\star_{2}^{9}$ is a semistar operation on $D$.

$\star_{3}^{9}$ is a semistar operation on $D$.

$\star_{4}^{9}$ is a semistar operation on $D$, and $\star_{4}^{9}=\mathrm{v}^{\prime}$.

(2.10) and (2.12) imply the following,

(2.13) Let $\mathcal{S}:=\left\{\star \in \Sigma^{\prime}(D) \mid D^{\star}=D\right\}$. Then $|\mathcal{S}|=25$. 
(2.14) $\quad\left|\Sigma^{\prime}(D)\right|=30$.

Proof. We have $|\Sigma(V)|=2$ and $|\Sigma(W)|=2$. Let $\Sigma(V)=\left\{\sigma_{1}, \sigma_{2}\right\}$, and let $\Sigma(W)=\left\{\sigma_{3}, \sigma_{4}\right\}$. Let $\left[\sigma_{1}\right]$ (resp., $\left.\left[\sigma_{2}\right]\right)$ be the canonical extension of $\sigma_{1}$ (resp., $\left.\sigma_{2}\right)$ to a semistar operation on $V$. $\left[\sigma_{1}\right]$ (resp., $\left[\sigma_{2}\right]$ ) canonically introduces a semistar operation $\star^{1}$ (resp., $\star^{2}$ ) on $D$. We define $\left[\sigma_{3}\right],\left[\sigma_{4}\right], \star^{3}, \star^{4}$ similarly. Let $\star$ be a semistar operation on $D$ such that $e \neq \star \notin \mathcal{S}$. Then $D^{\star}=V$ or $D^{\star}=W$, say $D^{\star}=V$. $\star$ canonically introduces a semistar operation $\alpha(\star)$ on $V$. Then $\alpha(\star)=\left[\sigma_{1}\right]$ or $\alpha(\star)=\left[\sigma_{2}\right]$, say $\alpha(\star)=\left[\sigma_{1}\right]$. For every $I \in \overline{\mathrm{F}}(D)$, we have $I^{\star^{1}}=(I V)^{\left[\sigma_{1}\right]}=(I V)^{\alpha(\star)}=(I V)^{\star}=$ $\left(I D^{\star}\right)^{\star}=I^{\star}$. Hence $\star^{1}=[\star]$, and hence we have $\Sigma^{\prime}(D)=\mathcal{S} \cup\left\{e, \star^{1}, \star^{2}, \star^{3}, \star^{4}\right\}$.

\section{REFERENCES}

[1] R. Matsuda, Note on the number of semistar operations, VIII, Math. J. Ibaraki Univ. 37 (2005), 53-79.

[2] R. Matsuda, The semistar operations on certain Prüfer domain, Math. J. Ibaraki Univ. 43 (2011), 1-12. 